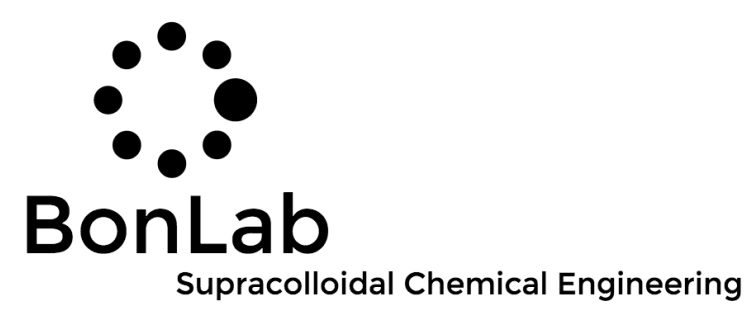

\title{
Thermotropic Icy Road Sign with Light Scattering and Fluorescence Response
}

Received 8th April 2021,

\author{
Joshua R. Booth, ${ }^{+a}$ Robert A. Young, ${ }^{+a}$ Andrés N. Richards Gonzales, ${ }^{a}$ Zachary J. Meakin, ${ }^{a}$ Corinna \\ M. Preuss-Weber, ${ }^{a, b}$ Ross W. Jaggers ${ }^{a}$ and Stefan A. F. Bon*a
}

\begin{abstract}
Prototypes of flexible, electricity-free, ice warning signs for roads and pavements have been developed. A thermotropic response in the form of an upper critical solution (UCST) type phase separation targeted near the freezing point of water manifests itself through light scattering as a clear-to-opaque transition. It is simultaneously amplified by an enhanced photoluminescence effect. The conceptual road sign application is a multi-lamellar flexible strip with an active layer of a polystyrene-based solution. The solvent is a plasticizer, here either dioctylphthalate (DOP) or its alternative 1,2-cyclohexane dicarboxylic acid diisononyl ester (DINCH). A collection of styrene-based macromolecules were made by free radical (co)polymerization, varying molecular weight and monomer feed composition. UCST type phase diagrams for the polymer solutions were constructed from cloud point data measured by a bespoke photographic set-up, in which up to 30 samples were measured simultaneously using both light scattering, that is opacity, and fluorescence. For the latter, the concept of restricted motion enhanced photoluminescence, often referred to as aggregation-induced emission (AIE), was used. Polystyrene labelled with tetraphenylethylene (TPE) was used for this. The contrast between 'ON' and 'OFF' states in the protoype ice warning signs was optimized by tuning the polymer concentration and the active layer thickness. Our prototype signs show full reversibility over many temperature cycles. We believe the concept can be of wider use in electricity-free signs and labels.
\end{abstract}

\section{Introduction}

At sub-zero temperatures (Celsius scale), the presence of ice poses a dangerous hidden risk to both pedestrians and road users. In the UK, in the last five years, 8390 road traffic accidents occurred in frosty or icy conditions. ${ }^{1}$ Ice warning systems currently in use are static signs that need to be physically placed or are complex and electrically powered. In the effort to move towards a sustainable future, there is increasing importance on warning indicators and sensors to respond autonomously to environmental change without the use of electricity.

The temperature-dependent transition from an optically transparent, single phase material into a phase separated analogue, is called thermotropy. The emerging phases commonly have different refractive indices, hereby triggering

\footnotetext{
a. Department of Chemistry, University of Warwick, Coventry, CV4 7AL, United Kingdom https://www.bonlab.info/

b. Department of Chemistry and Biotechnology, Darmstadt University of Applied Science, Stephanstr. 7, 64295 Darmstadt, Germany.

†J.R.B. and R.A.Y contributed equally. The manuscript was written through contributions by all authors. All authors have given approval to the final version of the manuscript.

*Email: s.bon@warwick.ac.uk

Electronic Supplementary Information (ESI) available: Video of prototype sign in use; experimental details, thermal imaging and example of analysis for the photographic plate reader; experimental details, molecular weight characterisation, cloud point curves, phase diagrams and critical phase conditions for styrene homopolymers and copolymers; characterisation of poly(styrene-co-4-(1,2,2triphenylvinyl)phenyl acrylate) (PS-co-TPEA); UV-Vis and fluorescence intensity spectra of PS-CO-TPEA/DINCH.
}

the scattering of light, so that the optical appearance of the material changes from clear to opaque. Beside a difference in refractive index, the geometry and size of the phase separated domains play their role in scattering. Polymer thermotropic materials which phase separate on heating are classified by a lower critical solution temperature (LCST), whereas systems that phase separate upon cooling have an upper critical solution temperature (UCST). The temperature at which the change in transparency from clear to opaque is observed for a particular material composition is often referred to as the cloud point. Phase diagrams can be determined experimentally for cloud point measurements against composition and predicted with thermodynamic models, ${ }^{2}$ a classical candidate being the FloryHuggins lattice-based theory. ${ }^{3,4}$ The LCST and the UCST are the minima and maxima on the could point curves, also referred to as phase co-existence curves.

A practical application of polymeric thermotropic materials is the regulation of solar radiation in buildings. ${ }^{5,6} \mathrm{In}$ recent times, these 'smart windows' have attracted considerable attention but can traced back as early as the 1950 s. $^{7}$ By autonomously and reversibly decreasing solar radiation in buildings at high temperatures, energy spent on building cooling is reduced. Polymeric materials used for thermotropic glazing include polymer blends, ${ }^{8,9}$ hydrogels, ${ }^{10-13}$ and polymerdispersed liquid crystal films ${ }^{14-17}$ The concept to use an LCST phase transition as a light reflector gate to prevent overheating, is also used in solar collectors. ${ }^{18}$ 
(a)

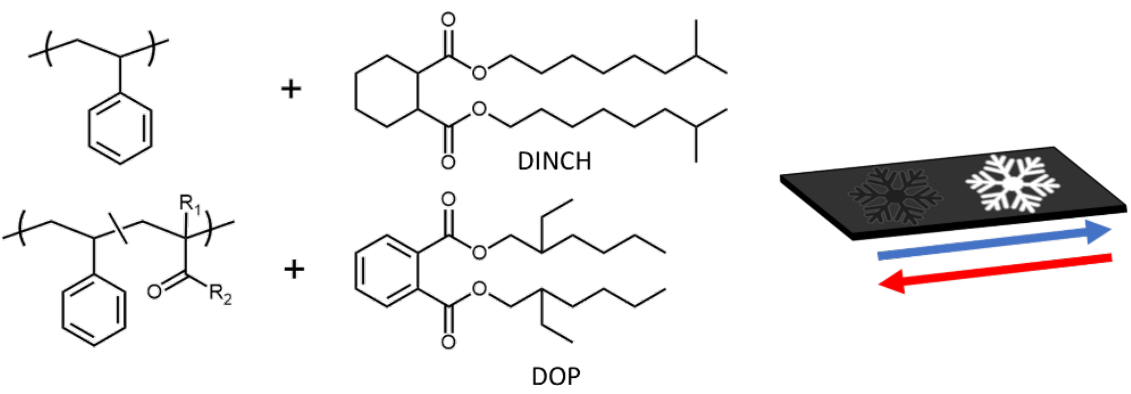

(b)<smiles>CC(CC(C)(C)C)C(C)(C)CC(C)(C)C(=O)Oc1ccc(C(=C(c2ccccc2)c2ccccc2)c2ccccc2)cc1</smiles>

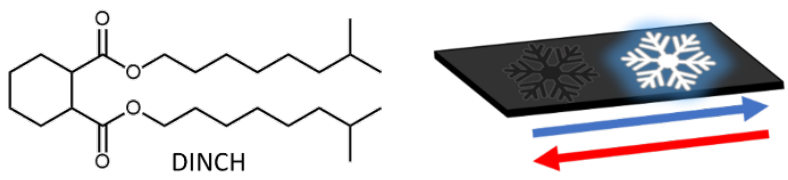

$\mathrm{P}(\mathrm{S}-\mathrm{Co}$-TPEA)

Scheme 1 The contrast swich ng temperature of the cy road $\mathbf{g}$ n can be tailored in two ways. (a) Opacty swich by ght scaltering: for use in 1, 2-cyclohexane d carboxy $c$ aca a sonony ester (D NCH), the mo ecu ar we ght of po vstyrene was varied For use n d octv terephtha ate (DOP) stvrene was codo vmer sed with a larety of monomers, ethy acrvate $\left(R_{1} H, R_{2} O_{2} H_{5}\right)$, 2-ethylhexyl acrylate $\left(R_{1} H, R_{2} O C_{8} H_{17}\right)$, ethyl methacrylate $\left(R_{1} C_{3}, R_{2} O C_{2} H_{5}\right)$, lauryl acrylate $\left(R_{1} H, R_{2} O C_{12} H_{25}\right)$, methyl methacrylate $\left(\mathrm{R}_{1} \mathrm{CH}_{3}, \mathrm{R}_{2} \mathrm{OCH}\right)$ and $\mathrm{N}$-isopropylacrylamide $\left(\mathrm{R}_{1} \mathrm{H}\right.$, $\left.\mathrm{R}_{2} \mathrm{NHC} \mathrm{C}_{3} \mathrm{H}_{7}\right)$. (b) Contrast swich by eht scatter ing and enhanced r vorescence: polystyrene labelled with 4-(1,2,2-triphenylvinyl)phenyl acrylate (TPEA) was used in DINCH.

Research into LCST-type polymer solutions and gels has been especially extensive for aqueous systems, ${ }^{19,20}$ with many thermotropic applications being used for reactive glazing. ${ }^{21}$ Combining LCST-type polymers with solvatochromic dyes also allows for use in a variety of sensors, as reviewed by Pietsch and coworkers. $^{22}$

Compared to triggering opacity when the temperature increases, the use of UCST-type polymers have received considerably less attention. The UCST phase behaviour of polymers has been studied in aqueous, ${ }^{23}$ water/alcohol, ${ }^{24}$ and organic solvents. ${ }^{25}$ Development of systems with optical applications in mind, however, have been sporadic. Seeboth and co-workers showed that hydrogel-based systems made from a blend of poly(vinyl alcohol) and ethoxylated polydimethylsiloxane, crosslinked with borax in water, went through opaque-translucent-opaque transitions, with translucency between room temperature and $55^{\circ} \mathrm{C} .{ }^{26}$ Danko et al. applied thermoresponsive hydrogels for use as a temperature indicator using zwitterionic copolymers. ${ }^{27}$ The opacity of the material was reversible, but there was a large volume difference between states. Volume contraction during phase separation of the material was overcome by Ding et al. with the combination of physical and chemical crosslinking for use in glazing. ${ }^{28}$ The opacity of their windows could also be regulated by electrical heating. As mentioned earlier, to reduce energy cost on cooling buildings in hot weather, LCST-type polymers are used in reactive glazing. Alternatively, a multilayer hydrogel coating, developed by La and co-workers, demonstrates the usefulness of UCST materials in colder conditions. ${ }^{13}$ Under opaque conditions, transmittance of mid-IR radiation was reduced, which they demonstrated on systems with a UCST between 25 and $55^{\circ} \mathrm{C}$. The applications for UCST hydrogels are naturally limited by the freezing point of water.
UCST polymer/alcohol systems can theoretically bring a positive outcome. One such system uses PMMA in water/ethanol. ${ }^{29}$ The inclusion of a chromatic dye allowed for accurate characterisation of the broad temperature sensing range and provided information on the globule-to-chain transition. The UCST was tuned between 29 and $47{ }^{\circ} \mathrm{C}$ depending on the water/ethanol ratio, a transition temperature too high for our intended application. We therefore decided to employ a nonaqueous UCST system.

We set out to design prototypes of flexible, electricity-free ice warning signs for roads and pavements and use a UCST thermotropic response near the freezing point of water. For use as an ice warning indicator, two types of prototype signs were developed. The first, illustrated in Scheme 1a contains an active layer composed of mixtures of either polystyrene (PS) or styrene copolymers with phthalate or non-phthalate plasticizer. Above the cloud point temperature, the mixture is transparent. The black base layer of the sign absorbs light and the snowflake symbol is not particularly visible. Lowering the temperature of the patch causes the polymer solution to separate, light is scattered and with the use of a top stencil layer, a white snowflake appears.

The phase separation behaviour of PS has been measured in a variety of organic solvents, such as acetone ${ }^{30}$, cyclic and aromatic solvents ${ }^{31}$, alkyl acetates ${ }^{32}$ and others ${ }^{33}$. However, for use in a warning indicator, the high volatility of these solvents is a major concern. Since the cloud point is a function of polymer/solvent composition, evaporation of the solvent would alter the switching temperature of the sign. To overcome this, dioctyl phthalate (DOP) and 1,2-cyclohexane dicarboxylic acid diisononyl ester (DINCH) were used. These solvents have reported vapour pressures of $1.3 \times 10^{-4}$ and $5.83 \times 10^{-4} \mathrm{~Pa}$ at $50^{\circ} \mathrm{C}$, respectively. ${ }^{34,35}$ The effect of molecular weight on the solubility 
of PS in DOP has been studied by Rangel-Nafaile and MuñozLara who showed, in line with theory, that the critical temperature for phase separation decreased for lower molecular weights. ${ }^{36}$ Stepanek et al. characterized the chain-toglobule transition of PS in DOP using light scattering techniques. ${ }^{37}$ Tao and co-workers investigated the effect of solvent size and found two routes for the collapse of polymer chains. ${ }^{38}$ In the 'middle-sized solvent', DOP, it was observed that PS forms single chain aggregates before macroscopic phase separation.

To enhance the effectiveness of the warning sign at night, a prototype sign that displays an increase in fluorescent emission at low temperatures, in addition the effect in opacity due to light scattering, was developed. This was achieved by copolymerising styrene with a monomer derived from the fluorophore, 1,1,2,2-tetraphenylethylene (TPE). As illustrated in Scheme 1b, at high temperatures, the polymer dissolves in the DINCH and the snowflake pattern is barely visible. TPE has a low fluorescence quantum yield when solvated due to its unique molecular structure. At temperatures below the cloud point, the solvated polymer chains collapse. A difference in polymer/solvent refractive index causes light to be scattered and a restriction in the intramolecular motions of TPE cause an increase in fluorescence intensity. With the use of a top stencil layer, a bright image appears in the dark under UV-light.

The phenomenon of molecules which display an increase in fluorescence from the solvated state to the 'solid state' can be traced back to the work of Jelley ${ }^{39}$ and Scheibe, ${ }^{42}$ in the 1930 s. $^{40}$ Both authors independently observed a spectral shift and fluorescence increase on dye aggregation, however the same effect can occur without intermolecular interactions. The 'solid state' which triggers the fluorescence increase can be generated by low temperatures and/or high viscosities, in matrixes that are amorphous or crystalline. The effect of viscosity and temperature on the fluorescence quantum yield of TPE ${ }^{41,42}$ and other molecules ${ }^{43-46}$ was studied between 19671990. In more recent years the phenomenon was popularised by Tang and co-workers with the term aggregated-induced emission (AIE). ${ }^{47,48}$ As the emission effect had already been shown to occur in the absence of aggregation, this term is somewhat ambiguous and later altered to include emission that occurs due to restriction of intermolecular motion (RIM), which includes rotation (RIR) or vibration (RIV). ${ }^{49}$ Studies of AIE type molecules have been expanded to polymeric species. ${ }^{50}$ The study of thermotropic AIE polymers was reported by Tang et al. who copolymerised a monomeric TPE derivative with NIPAM. ${ }^{51}$ Many more iterations of thermoresponsive TPE copolymers have been studied, which include combinations with PNIPAM, ${ }^{52-54}$ polyethylene glycol $^{55}$ and polyacrylamide hydrogels. ${ }^{56}$ Water-based thermoresponsive polymers that exhibit a UCST phase transition have recently been reported by Jia et al. ${ }^{57}$ An increase in fluorescence was observed on cooling and the process was visualised using confocal microscopy.

In this work we set out to combine the thermodynamic study of polystyrene-based solutions at low temperatures with an ice warning indicator that has the potential to improve road safety in a simple, inexpensive, electricity-free approach. To our knowledge the phase separation of PS copolymers in DOP and homopolymer PS in DINCH has not yet been examined. Therefore, we firstly investigated the temperature dependant solubility of PS copolymers in DOP and a range of PS molecular weights in DINCH. Due to the considerable number of polymer/solvent combinations required to produce phase diagrams, a custom photographic plate reader was developed to measurement up to 30 samples in parallel. The results were used to optimise the design of the prototype thermotropic ice warning signs, which not only showed a UCST-type response in the form of light scattering but also through enhanced fluorescence upon phase separation.

\section{Experimental}

\section{Materials}

Acryloyl chloride ( $\geq 96 \%$ ) was purchased from Alfa Aesar. 1,2Cyclohexane dicarboxylic acid diisononyl ester (Hexamoll ${ }^{\circledR}$ DINCH) was kindly supplied by BASF. 1-(4-hydroxyphenyl)-1,2,2triphenylethylene (TPE-OH, 99.75\%) was purchased from Chemscene. Magnesium sulfate, methanol $(\geq 99.9 \%)$, sodium hydroxide $(\mathrm{NaOH})$, tetrahydrofuran (THF, $\geq 99 \%)$, triethylamine (TEA, $\geq 99 \%$ ) and xylene (analytical grade) were purchase from Fisher Scientific. Dichloromethane (DCM, anhydrous, $\geq 96 \%$ ), dioctyl phthalate (DOP, $\geq 96 \%$ ), ethyl acrylate, (EA, 99\%), 2ethylhexyl acrylate (2EHA, 98\%), ethyl methacrylate (EMA, 99\%), lauryl acrylate (LA, 90\%), methyl methacrylate (MMA, 99\%), N-isopropylacrylamide (NIPAM, 99\%), oil blue (96\%), 4phenylazophenol, rose bengal, sodium bicarbonate $(\geq 99.5 \%)$, styrene (99\%) and tetrahydrofuran (inhibitor-free, $\geq 99.9 \%$ ) were purchased from Sigma-Aldrich. Double sided adhesive, acetate sheets ( $2.7 \mathrm{~mm}$ thick) and printable acetate sheets were purchased from Stix2.

\section{Synthesis of polystyrene copolymers by bulk polymerisation}

Styrene and comonomers, in accordance with Table S1, were filtered through basic alumina to remove inhibitors and purged with nitrogen gas. Initiation was induced by submerging the vials into an oil bath at $140{ }^{\circ} \mathrm{C}$. After 1 hour, the reactions were quenched with air and cooled in an ice bath. The resulting solutions were diluted with THF before precipitating into cold methanol. The polymer was then dried in a vacuum oven before characterisation.

\section{Synthesis of polystyrene by solution polymerisation}

Styrene was filtered through a column of basic alumina and added to varying amounts of xylene, outlined in Table S2. The solutions were purged with nitrogen gas and the reaction was commenced by submerging the vials in an oil bath at $135{ }^{\circ} \mathrm{C}$. After 3 hours the reactions were quenched with air and cooled in an ice bath. The polymer solutions were diluted with THF before being precipitated into cold methanol. The polymer was then dried in a vacuum oven at $100{ }^{\circ} \mathrm{C}$ before characterisation. 


\section{Synthesis of 4-(1,2,2-triphenylvinyl)phenyl acrylate}

Following a procedure reported by Qian Li et al., $58 \mathrm{TPE}-\mathrm{OH}(0.25$ $\mathrm{g}, 0.716 \mathrm{mmol})$ and TEA $(0.15 \mathrm{~mL}, 1.076 \mathrm{mmol})$ were dissolved in anhydrous DCM (35 mL). Such an excess of solvent was used as TPE-OH was sparingly soluble. Acryloyl chloride $(0.085 \mathrm{~mL}$, $1.05 \mathrm{mmol}$ ) was dissolved in DCM $(5 \mathrm{~mL})$ and added dropwise to the TPE-OH solution at $0{ }^{\circ} \mathrm{C}$. The reaction was stirrer at $0{ }^{\circ} \mathrm{C}$ for 30 minutes then at room temperature for 2 hours. The precipitate was removed by gravity filtration and the mixture was washed twice with saturated aqueous $\mathrm{NaHCO}_{3}$, twice with $\mathrm{pH} 9$ aqueous $\mathrm{NaOH}$ and twice with deionized water. The solvent was dried with magnesium sulfate and evaporated under reduced pressure, giving the desired product as a yellow solid $0.1052 \mathrm{~g}$ (yield $33.7 \%$ ) ${ }^{1} \mathrm{H}$ NMR $\left(400 \mathrm{MHz}, \mathrm{CDCl}_{3}\right)$ ס: $7.16-$ $6.99(\mathrm{~m}, 17 \mathrm{H}), 6.88(\mathrm{~d}, 2 \mathrm{H}, \mathrm{J}=8.56 \mathrm{~Hz}), 6.56(\mathrm{dd}, \mathrm{J}=17.24,0.98$ $\mathrm{Hz}, 1 \mathrm{H}), 6.27$ (dd, J = 17.24, $10.39 \mathrm{~Hz}, 1 \mathrm{H}), 5.97$ (dd, J = 10.51, $0.98 \mathrm{~Hz}, 1 \mathrm{H})$.

\section{Synthesis poly(styrene-co-4-(1,2,2-triphenylvinyl)phenyl acrylate)}

To a 3-neck RBF with a condenser fitted, 4-(1,2,2triphenylvinyl)phenyl acrylate $\left(3.306 \mathrm{mg}, 6.97 \times 10^{-3} \mathrm{mmol}\right.$ ) was added and the vessel purged with nitrogen gas for 1 hour. Separate stocks of styrene, with inhibitor removed, and xylene were prepared and purged with nitrogen gas for 30 minutes. After purging, styrene $(8.8 \mathrm{~mL}, 76.81 \mathrm{mmol})$ and xylene (13.39 $\mathrm{mL}, 113.04 \mathrm{mmol}$ ) were added to the RBF. The mixture was heated at $135{ }^{\circ} \mathrm{C}$ for 3 hours with stirring. The mixture was cooled and air was bubbled into the mixture to quench the polymerisation reaction. The polymer was precipitated into room temperature pentane and washed twice in cold pentane. The polymer was collected by vacuum filtration and dried under vacuum at $100^{\circ} \mathrm{C}$.

\section{Photographic plate reader}

To conduct the phase separation experiments, an aluminium plate with 36 wells (volume $0.25 \mathrm{~mL}$ ) was used. Its temperature was regulated by a thermoelectric cooler. Each well was fitted with a circle of black card on the base to aid with contrast During the measurement, the plate was covered with an antireflective coated glass slide to prevent solvent evaporation and ice formation on the plate. A single well was filled with pure solvent for temperature measurement. The aluminium plate and thermoelectric module were placed inside an open top box with cut-outs on either side covered in a non-woven fabric interfacing that acted as an optical diffuser. The plate was lit with two 17W LED globe blubs and a DLSR camera was positioned directly above using a tripod. To prevent the interference of ambient light, four retort stands were placed around the open-topped box and covered with black cloth. The samples were cooled from 20 to $-20{ }^{\circ} \mathrm{C}$ at a rate of $0.2{ }^{\circ} \mathrm{C} \mathrm{min}-1$. The phase transitions of the samples were determined using photographs taken during the experiment, the colour images were converted to grey scale and the average grey value for each well was determined as function of temperature. Further details about the technique and measurement procedure are provided in the supporting information.

\section{Measurement of road sign photoluminescence effect}

Prototypes signs containing poly(styrene-co-4-(1,2,2triphenylvinyl)phenyl acrylate) dissolved in DINCH were placed on a thermoelectric cooler located beneath a UVP UVGL-55 UV lamp with wavelengths of 254 and $365 \mathrm{~nm}$. A DLSR camera was positioned directly above using a tripod. A UV blocking filter was position in front the camera lens. The manufacturer of the UV filter specified $<0.5 \%$ transmittance below $410 \mathrm{~nm}$ and $>97 \%$ above $445 \mathrm{~nm}$. During the measurement, the thermoelectric plate was cooled from 30 to $-20^{\circ} \mathrm{C}$ at $0.2{ }^{\circ} \mathrm{C} \mathrm{min}^{-1}$. Photos were taken automatically every 60 seconds, the camera's shutter speed, aperture, ISO and white balance were fixed throughout. Ice formation below $0{ }^{\circ} \mathrm{C}$ was prevented by purging with nitrogen gas. Ambient light was regulated with a cover of black cloth over the instrument. For analysis of luminescence, the images were converted to 32-bit grey scale and the average grey value was determined with ImageJ software.

\section{Characterisation}

${ }^{1} \mathrm{H}$ NMR experiments were conducted on a Bruker Avance III HD $400 \mathrm{MHz}$ instrument. Chloroform-d was purchased from SigmaAldrich. Spectra were analysed using ACD laboratories software. Gel permeation chromatography was carried out using an Agilent Infinity II MDS instrumentation equipped with differential refractive index, viscometry, dual angle light scatter, and multiple wavelength UV detectors was used. The system was equipped with $2 \times$ PLgel Mixed C columns $(300 \times 7.5 \mathrm{~mm})$ and a PLgel $5 \mu \mathrm{m}$ guard column. THF with $0.01 \%$ butylated hydroxytoluene additive was used as the eluent. Samples were run at $1 \mathrm{~mL} \mathrm{~min}-1$ at $30^{\circ} \mathrm{C}$. PMMA and PS standards (Agilent EasyVials) were used for calibration. Analyte samples were filtered through a GVHP membrane with $0.22 \mu \mathrm{m}$ pore size before injection. Experimental molar mass $\left(M_{n}, S E C\right)$ and dispersity $(\bigoplus)$ values of synthesised polymers were determined by conventional calibration against PMMA and PS standards using Agilent GPC/SEC software. The Mark-Houwink-Sakurada parameters used for the PMMA standards were $\mathrm{K}=9.44 \times 10^{-3}$ $\mathrm{mL} \mathrm{g}^{-1}$ and $\alpha=0.719$, and for PS K $=14.1 \times 10^{-3} \mathrm{~mL} \mathrm{~g}^{-1}$ and $\alpha=$ 0.700. UV-Vis spectra were obtained using an Agilent Cary 60 UV-Vis spectrophotometer. Quartz cuvettes of $1 \mathrm{~cm}$ pathlength were used and the sample temperature was maintained at 25 ${ }^{\circ} \mathrm{C}$ using a thermostated single cell holder. Photoluminescence spectra were recorded on an Agilent Cary Eclipse fluorescence spectrophotometer. Fluorescence specific quartz cuvettes of 1 $\mathrm{cm}$ pathlength were used and the sample temperature was regulated with a Peltier block and multicell holder. The spectrophotometer is equipped with a $15 \mathrm{~W}$ xenon pulse lamp, with pulse width 2-3 $\mu$ s and power $64 \mathrm{~kW}$. For experiments conducted below $0{ }^{\circ} \mathrm{C}$, the measurement chamber was purged with nitrogen gas. Transmittance measurements were conducted on an Anton Paar Litesizer 500. The light source was semiconductor laser diode with power $40 \mathrm{~mW}$ and wavelength of $658 \mathrm{~nm}$. 


\section{Results \& discussion}

\section{Photographic plate reader and cloud point determination}

To investigate the significance of PS molecular weight and copolymer composition on the phase transition temperature, a matrix of mixtures was required. Thermally induced phase separations are typically recorded by spectrometers, by measuring a decrease in light transmittance through the sample. For an accurate measurement of polymer solution cloud points, a low rate of cooling/heating is required, to measure each polymer-solvent combination consecutively would be time consuming. Herein we devised an efficient and accurate method using a bespoke metal plate reader to record the phase transitions of up to 30 samples in parallel (Scheme 2). During the measurements, the plate's temperature was controlled by a thermoelectric cooler/heater. The sample temperature was accurately measured using a probe emersed in a well containing a polymer-free sample of DOP or DINCH. A consistent temperature across the plate was confirmed by infrared photography (Figure S1). Images of the plate were taken during the experiment, recorded in RAW format and processed into 32-bit grey scale. For each image, the mean grey value (G) was calculated for a 50 pixels diameter circle in the centre of each well (Figure S2). Each well has a black coloured base, seen through the transparent, miscible liquid at high temperatures. As the mixture phase separates it begins to scatter light and the mean grey value increases. From the image analysis a plot of $\mathrm{G} / \mathrm{G}_{0}$ is produced, where $\mathrm{G}_{0}$ is the lowest recorded mean grey value. As shown in Figure 1a the measurement of $10 \% \mathrm{w} / \mathrm{w}$ PS in DOP using the photographic method fits very closely to transmittance data recorded on a UV-spectrometer. Furthermore, because the temperature of the plate reader is cooled thermoelectrically, temperatures as low as $-20^{\circ} \mathrm{C}$ can be

(a)

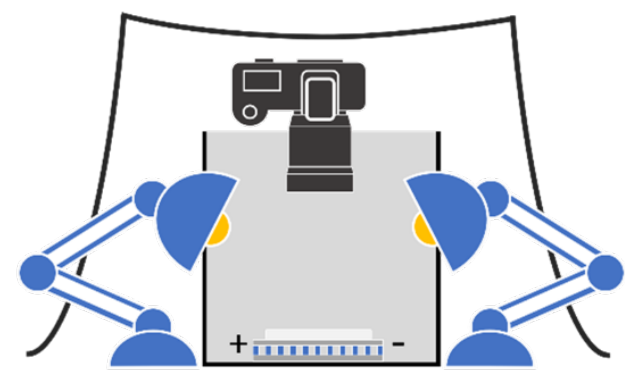

(b)

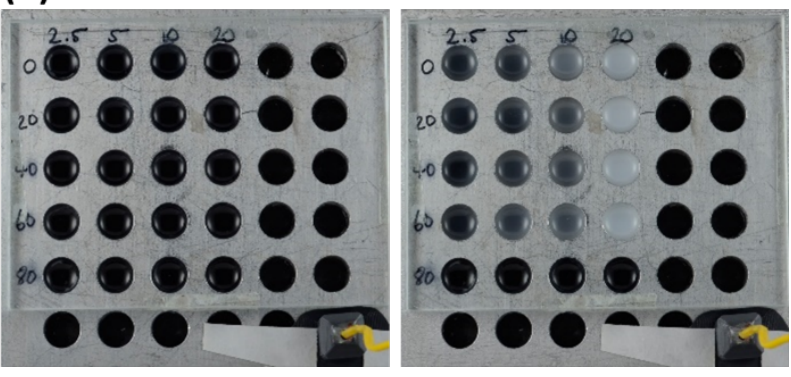

Scheme 2 (a) ustrat on of custom photograph c $p$ ate reader $A 36$ we p ate

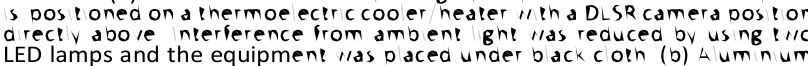

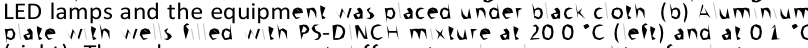
(right). The columns represent a fferent moecuar we ghts of povstvrene, whereas the rows represent different polymer-solvent ratios. measured easily. An example of the data analysis performed to obtain the cloud point is shown in Figure 1b. Firstly, a Boltzmann sigmodal function was applied until the fit converged. A tangent line was extended from inflection point of sigmodal fit and the intersect of this line with the baseline $(y=1)$ was determined as the cloud point, $T_{c p}$.

\section{Phase separation behaviour of polystyrene in phthalate and non-} phthalate plasticizers

Adjustability of the thermochromic switching temperature is key to the wider applications of the warning sign. The FloryHuggins theory for the phase behaviour for polymer solutions shows that the critical temperature of mixing a polymer solution is dependent on the enthalpic polymer/solvent compatibility as well as polymer molecular weight and composition (volume fraction of polymer in solution). ${ }^{59}$

The experimental value for the UCST of PS of high molar mass in DOP ( $300 \mathrm{~kg} \cdot \mathrm{mol}^{-1}$ ) was determined to be $7.6^{\circ} \mathrm{C}$ and in excellent agreement with the literature value of $7.4{ }^{\circ} \mathrm{C}$ for 280 $\mathrm{kg} . \mathrm{mol}^{-1}$ polystyrene. ${ }^{36}$ To investigate the effect of polymer/DOP compatibility, styrene was copolymerised with either ethyl acrylate (EA), 2-ethylhexyl acrylate (2EHA), ethyl methacrylate (EMA), lauryl acrylate (LA), methyl methacrylate (MMA) or $\mathrm{N}$-isopropylacrylamide (NIPAM). Each of these styrene comonomer combinations were synthesised over a range of feed ratios $0.5-10$ weight percent of comonomer (polymer characteristics listed in Table S3). Each copolymer at a given copolymer composition was mixed with DOP in 6 different polymer-solvent weight fractions. Owing to the high throughput of the photographic plate reader, analysis of 236 PS-copolymersolvent combinations was carried out. Cloud points for all combinations were calculated using the method described previously for Figure $1 \mathrm{~b}$ (examples for $\mathrm{P}(\mathrm{S}$-co-MMA) copolymers shown in Figures S3). Using the cloud points, phase diagrams for each copolymer series were produced (Figures S4) and the cloud point data was fit with a second order polynomial. The maximum was the experimentally determined UCST (Table S4). As shown in Figure 1c, increasing the mole fraction of comonomer in the styrene copolymer reduces the critical temperature in all cases. This is due to a more favourable polymer-solvent interaction. An interesting observation is that the drop in UCST is considerable, but less steep for those monomers that are more polar in nature. From the data in can be concluded that a tailored polymer can be made for a warning sign with a specific transition temperature.

The low melting point and vapour pressure of DOP meets the requirements for use in a temperature indicator, however there are known safety concerns regarding phthalate solvents. ${ }^{60,61}$ To negate these health risks, DINCH was used. DINCH has been manufactured as a direct replacement for DOP so shares similar properties such a high vapour pressure and low melting point. As well as being a less harmful solvent, the refractive index of DICNH, $\mathrm{n}_{\mathrm{D}}{ }^{20} 1.461$, is slightly lower than DOP, $\mathrm{n}_{\mathrm{D}}{ }^{20}$ 1.488. This works in our favour as larger refractive index gap between polymer and solvent, when phase separated, 

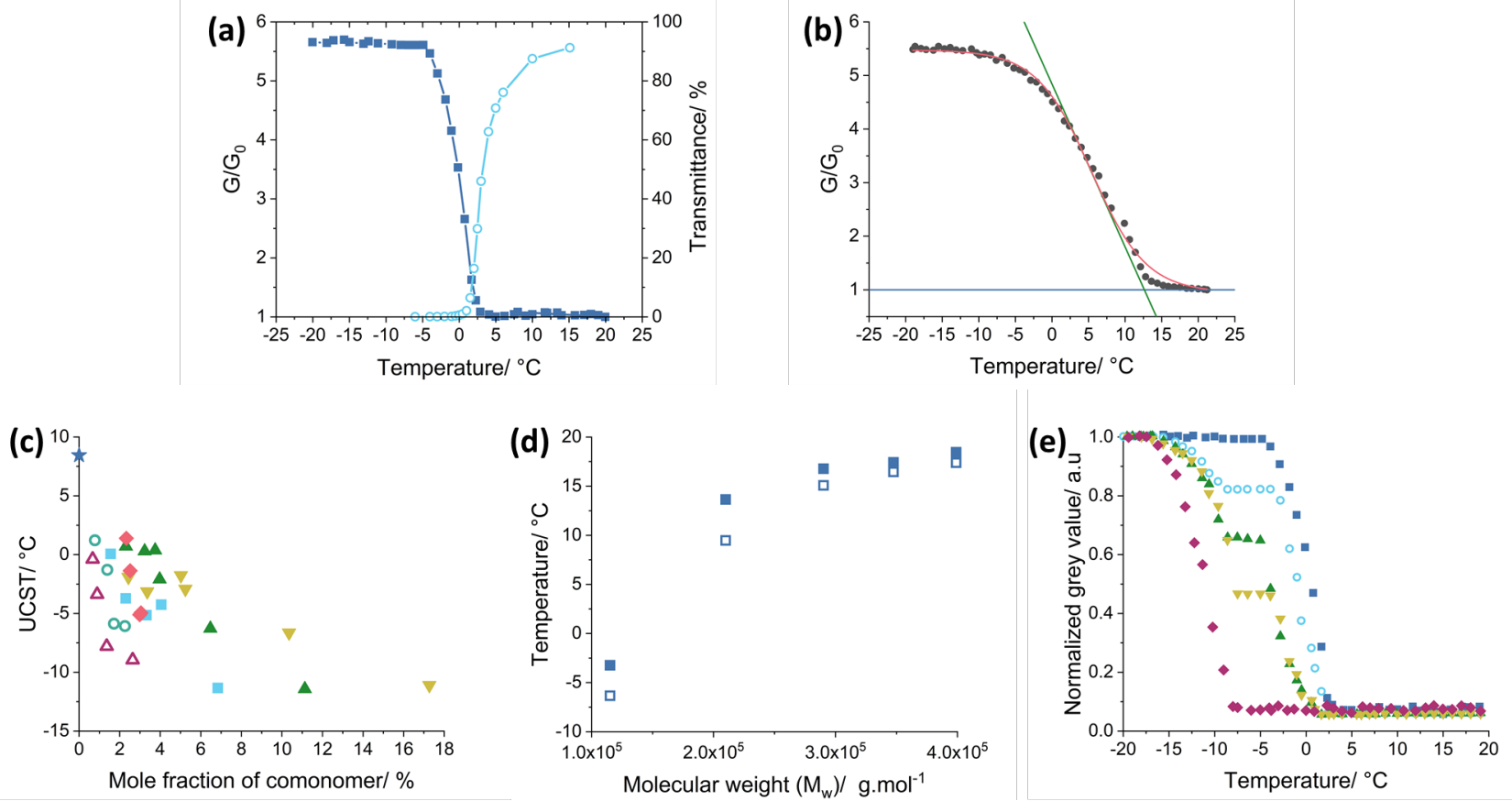

Figure 1 (a) Phase change response of polystyrene $\left(\mathrm{M}_{\mathrm{w}} 280,000 \mathrm{~g} \cdot \mathrm{mol}^{-1} \mathrm{\oplus} 3.0\right.$ ) in dioctyl phthalate (DOP) at $10 \% \mathrm{w} / \mathrm{w}$, measured by photographic plate reader technique (dark blue squares) and using UV-Vis spectroscopy at wavelength of $500 \mathrm{~nm}$ (turquoise open circles). (b) Cloud point determination from phase change curves measured using photographic technique of polystyrene dissolved in 2-cyclohexane dicarboxylic acid diisononyl ester (DINCH) Cloud points were determined at the intersection of a tangent line (green) from the centre of a Boltzmann sigmodal fit (pink) with the baseline (blue). (c) The change in upper critical solution temperature (UCST) of polystyrene copolymers in DOP as a function of comonomer mole fraction. Polystyrene (dark blue star), poly(ethyl methacrylate-co-styrene (light blue squares), poly(2-ethylhexyl acrylate-co-styrene) (turquois open circles), poly(ethyl acrylate-co-styrene) (green triangles), poly (lauryl acrylate-co-styrene

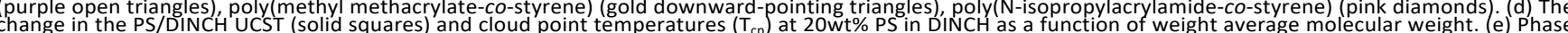
change response of polymer blends in DOP, blends of polystyrene and poly(styrene-co-lauryl acrylate) were mixed by weight ratio. Polystyrene (dark blue squares) 75:25 PS:P(S-co-LA) (light blue open circles), 50:50 PS:P(S-co-LA) (green triangles), 75:25 PS:P(S-co-LA) (gold triangle) and P(S-co-LA) (purple diamonds).

produces more scattering and greater contrast between OFF and ON states.

PS was synthesised by bulk and solution polymerisation, with molecular weight regulated through control of monomer to solvent ratio (Table S5). Combinations of PS in DINCH 1-20\% $\mathrm{w} / \mathrm{w}$ were prepared and the photographic plate reader was used to record the phase transitions. Cloud points were determined from plots of $\mathrm{G} / \mathrm{G}_{0}$ against temperature (Figure $\mathrm{S} 5$ ) and phase diagrams were produced (Figure S6). From each PS phase diagram, the UCST was experimentally determined as the highest $y$-axis value, using a polynomial fit (Table S6). Figure $1 \mathrm{~d}$ illustrates the ability to tune the temperature at which phase separation of the mixture occurs. The PS/DINCH UCST, together with the cloud points at a polymer/solvent weight fraction of 0.2 , are plotted as a function of molecular weight. A marked drop in the phase separation temperature as molecular weight decreases is characteristic of UCST behaviour, and indeed has also been observed for PS in cyclic alkanes and acetates. ${ }^{31,32}$ The weight average molecular weight was chosen to represent the polymer molecular weight, due to the dispersity being between 2.0-2.6, typical for free radical polymerisations. It has been shown that for disperse samples the higher weight fractions have a greater influence on the cloud point. ${ }^{62,63}$

This set us to explore the phase separation of binary polymer blends in DOP, where we used mixtures of two polymers of different chemical composition, and thus different cloud points. The idea was to incrementally regulate the level of opacity. The results are shown in Figure 1e. The phase change curves of three $10 \mathrm{wt} \%$ blends of PS ( $\mathrm{M}_{\mathrm{w}} 280,000 \mathrm{~g} . \mathrm{mol}^{-1}, \oplus 3.0$ ) and $\mathrm{P}(\mathrm{S}-\mathrm{Co}-\mathrm{LA})\left(\mathrm{M}_{\mathrm{w}} 384,000 \mathrm{~g} \cdot \mathrm{mol}^{-1}, \mathrm{\oplus} 1.94,2.6 \mathrm{~mol} \%\right.$ of LA in the random copolymer) mixtures, as well as the respective pure polymer solutions in DOP, are shown. The cloud points of pure $\mathrm{PS}$ and $\mathrm{P}(\mathrm{S}-\mathrm{CO}-\mathrm{LA})$ polymers were determined to be 2 and -8.5 ${ }^{\circ} \mathrm{C}$, respectively. Due to the large difference in $T_{c p}$ of the pure polymers, the 75:25, 50:50 and 25:75 PS:P(S-co-LA) weight ratio blends display two cloud points with a plateau region for the normalized grey value in-between. The lower and higher $T_{c p}$ of the three blends are in line with the respective pure polymers $T_{c p}$, slight variation accounted for a result of the difference in weight fraction. Blends with a higher concentration of PS reached a higher grey value plateau, indeed demonstrating that a two-stage stepwise control of opacity is possible.

The phase diagram plots of Figures S4 and S6 illustrate that cloud points can be tuned by copolymer composition and molecular weight, in addition to the volume or weight fraction of polymer in solution. For DOP or DINCH mixtures, the greatest opacity of a sample, measured as $G / G_{0}$, reached at $T \ll<T_{c p}$, was recorded in samples with the highest polymer weight fraction of 0.2. This influenced our choice when designing effective ice warning sign prototypes.

\section{Design and performance of thermotropic sign}

For use as an ice warning system, $5 \times 5 \mathrm{~cm}$ prototypes were built and tested. Illustrated in Figure $2 c$, the thermotropic patches were made of three cellulose acetate sheets bound together with layers of pressure sensitive adhesive. Cellulose acetate was selected as it has a high optical transmittance as well as good mechanical and thermal properties. The base acetate sheet is 

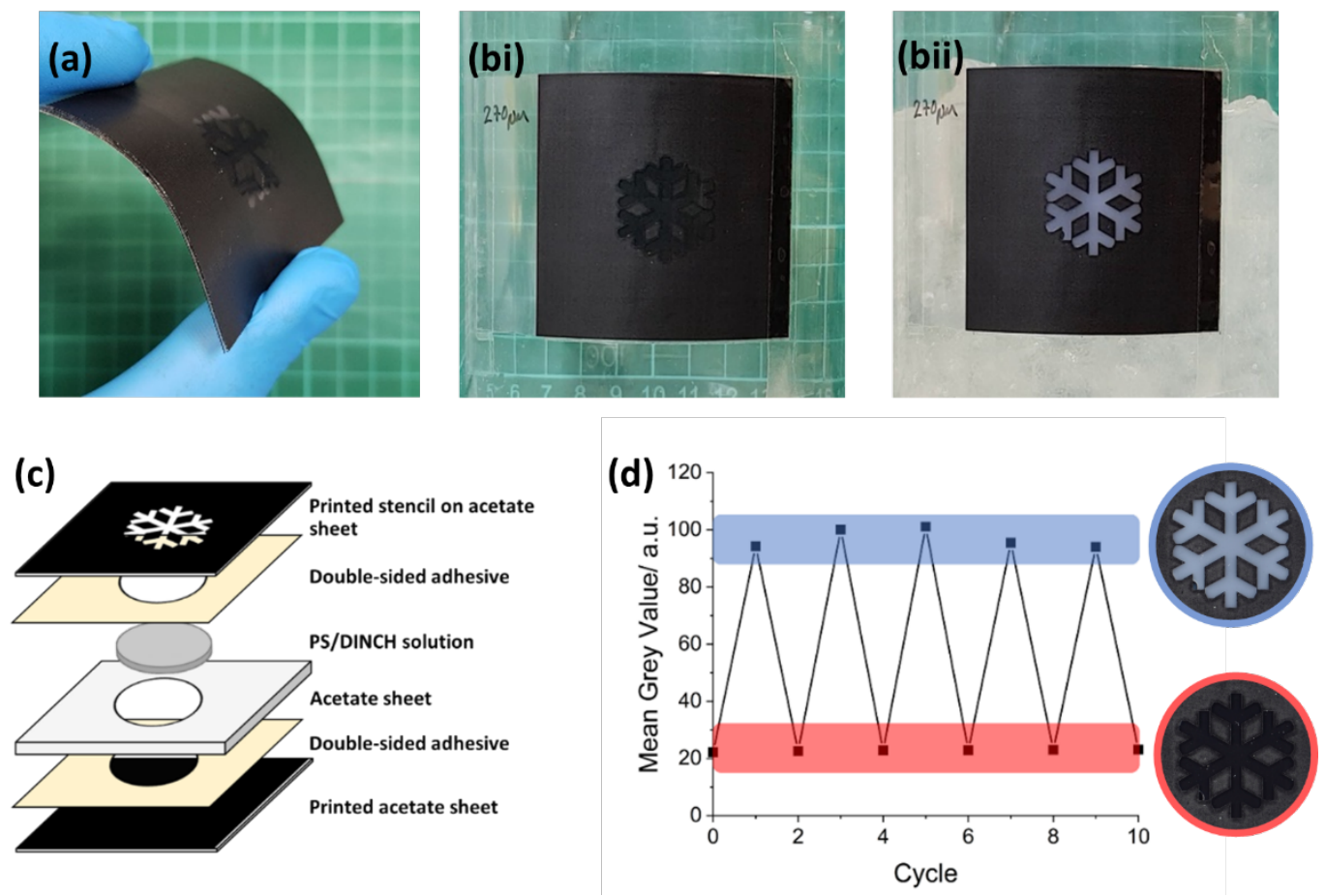

Figure 2 (a) Th $n$ aver patch can be fexed and app ed to curved surfaces r requ red wh st ma nta n ng fu funct on (b) Frames taken from video (found in ESI) of patch adhered to gass beaker at room temperature () and conta $n \mathrm{ng}$ ce water for 60 seconds (i). (c) Exploded view diagram showing lamella structure of ice warning

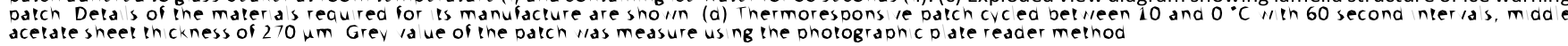

black to maximise contrast between OFF and ON states. The central sheet has a circular section removed, which is filled with the PS/DINCH mixture. The PS used had a $M_{w}$ of $210 \mathrm{~kg} \mathrm{~mol}^{-1}$ (PS60\%XY, Table S5, Figure S5d) and was dissolved in DINCH at weight fraction of 0.2 . This specific molecular weight and polymer/solvent composition was chosen to maximise scattering with a cloud point close to $0{ }^{\circ} \mathrm{C}$. Mixtures with greater amounts of PS (at higher molar mass to have the same transition temperature) were not suitable to use in sign manufacturing, due to the high viscosity of the mixtures. The top sheet of the patch has a customisable printed stencil which causes a snowflake symbol to appear when cooled below the switching temperature. The thickness of sign's central sheet, either 100 or $270 \mu \mathrm{m}$, could be altered to favour flexibility or contrast. It was found that the increased pathlength of the liquid in the $270 \mu \mathrm{m}$ patch improved contrast by $15 \%$ without impairing flexibility.

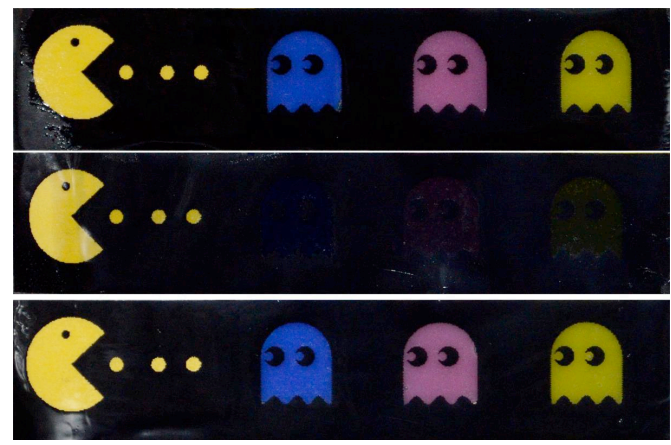

Figure 3 Patch d sp ay $\mathrm{ng}_{\mathrm{g}} \mathrm{u}$ co our temperature response by add $\mathrm{ng}$ o b ue, rose bengal, and 4-ohenv azopheon dves to po vstyrene-DOP mixture. The patch contan $n g$ PS ( $M W 280,000 \mathrm{~g}$ mo-1 $\mathrm{D} 30$ ) $n$ DOP at $10 \mathrm{wt} \%$ was maged
at be ow, above and be $0 w$ phase separat on temperature, too made and bottom, respectively.
Figure $2 \mathrm{a}$ illustrates the flexibility of the $270 \mu \mathrm{m}$ patch (see also the video in ESI). The patch is bent in each direction before being placed onto a thermoelectric plate set to $0{ }^{\circ} \mathrm{C}$. Due to the time taken for the patch's shape to relax, faster switching times were achieved when the material was flattened and in close contact with the surface. The flexibility of the patch allows it to function on curved surfaces. This is demonstrated with a glass beaker and ice water, with frames from the video are shown in Figure 2(bi) and (bii). Maximum contrast from OFF to ON state was reached in approximately 60 seconds with the $270 \mu \mathrm{m}$ patch producing the greatest contrast.

The reversibility of the signs was analysed using photographic grey value method. Both signs show consistent contrast values across 10 cycles between 10 to $0{ }^{\circ} \mathrm{C}$ with 60 second intervals between temperature changes. Reversibility for the $270 \mu \mathrm{m}$ and $100 \mu \mathrm{m}$ sign is demonstrated in Figures $2 \mathrm{~d}$ and $\mathrm{S7}$, respectively.

As well as a black to white transition, a full colour response can be achieved using dyes. Demonstrated in Figure 3 , an easily recognisable video game scene was enacted using three dyes (oil blue, rose bengal, and 4-phenylazophenol, 10 mg) which dissolved in the PS-DOP mixture at all temperatures. Below the thermally induced phase transition, the PS rich phase acts as an opacifier. Heating the patch causes the PS to dissolve, through the transparent mixture the black background absorbed light and the colours are barely visible.

\section{Combination of phase separation and aggregation-induced} emission

The thermotropic temperature response of the PS/DINCH sign was enhanced by the incorporation of an AIE monomer. 4(1,2,2-triphenylvinyl)phenyl acrylate) (TPEA) was synthesised by 
a method adapted from by Qian et al. ${ }^{58}$ and characterised by ${ }^{1} \mathrm{H}$ NMR (Figure S8). Poly(styrene-co-4-(1,2,2-triphenylvinyl)phenyl acrylate) (P(S-CO-TPEA)) was synthesised by solution polymerisation. The copolymer had a molar ratio of TPEA:S 1:4600 and a weight average molecular weight of 210,000 g. mol-1 (Figure S9). Only a very low concentration of TPEA in the copolymer was required as a high concentration of polymer in DINCH (20\%w/w, as before) is necessary for optimal contrast between OFF and ON states. Too much TPEA may have a pronounced effect on the solubility characteristics, in line with what we observed with other comonomers (see Figure 1c). The amount of TPEA incorporated into P(S-Co-TPEA) was compared to the monomer feed molar ratio using a UV absorbance calibration curve (Figure S10). The experimental molar ratio was found to be 1:4600 TPEA:S compared to the feed ratio of $1: 11,000$. This deviation is thought to be due to composition drift during radical polymerisation. As the polymer was synthesised to low monomer conversion, composition drift towards TPEA most likely occurred, evidenced by literature reactivity ratios of similar acrylate comonomers. ${ }^{64,65} \mathrm{~A}$ molecular weight of $210,000 \mathrm{~g} . \mathrm{mol}^{-1}$ was targeted to obtain a polymer with a UCST in DINCH of $13{ }^{\circ} \mathrm{C}$ and the molecular weight was confirmed by GPC analysis (Figure S9). The P(S-coTPEA) was dissolved in DINCH at $10 \% \mathrm{w} / \mathrm{w}$, targeting a cloud point of $10{ }^{\circ} \mathrm{C}$. This transition temperature was chosen to allow for the analysis of phase separation and fluorescence intensity (FI) down to 30 degrees below $T_{\mathrm{cp}}$. A temperature of $-20^{\circ} \mathrm{C}$ was the lowest that could be measured with the available equipment. Note that a P(S-Co-TPEA)/DINCH solution of $20 \%$ $\mathrm{w} / \mathrm{w}$ would have a transition temperature a few degrees lower, and an approximate $40-50 \%$ increase in contrast.

The phase separation of the $10 \% \mathrm{w} / \mathrm{w}$ P(S-Co-TPEA)/DINCH solution was characterised by measuring light transmittance with a light scattering detector, using a $1 \times 1 \mathrm{~cm}$ optical glass cuvette. A laser wavelength above the absorbance of TPEA was used so that transmitted light was only a function of opacity. In Figure 4a, light blue open circles, a decrease in light transmission through the sample from 15 to $10{ }^{\circ} \mathrm{C}$ is observed due to phase separation. The sharp transition to $0 \%$ transmittance below $10^{\circ} \mathrm{C}$ is due to the strong opacity of the sample and relatively long path length compared to the 0.27 $\mathrm{mm}$ layer of liquid used in the prototype sign.

Trying to monitor the light emission response with a conventional fluorospectrometer leads to complications in data interpretation as a result of light scattering from the phase separating polymer. Due to the large decrease in transmittance of the 10 wt\% mixture, a drop in fluorescence intensity (FI) is observed at the onset of phase separation when measured at a detector angle of $90^{\circ}$ (Figure S11). This effect was also observed for phase separated PNIPAM-TPE copolymers in water, ${ }^{51}$ as well as solvatochromic dyes in water66 or ethanol. ${ }^{29}$ It can be overcome by measuring at much lower polymer concentrations ( $\left.<0.1 \mathrm{mg} \cdot \mathrm{mL}^{-1}\right)$ so that scattering is minimized. ${ }^{52,53}$ However, doing so alters the cloud point temperature drastically.

We therefore modified our plate reader set up to measure the luminescence of the $\mathrm{P}(\mathrm{S}-\mathrm{Co}-\mathrm{TPEA}) / \mathrm{DINCH}$ mixture as a function of temperature. For this we exposed the sample to UV

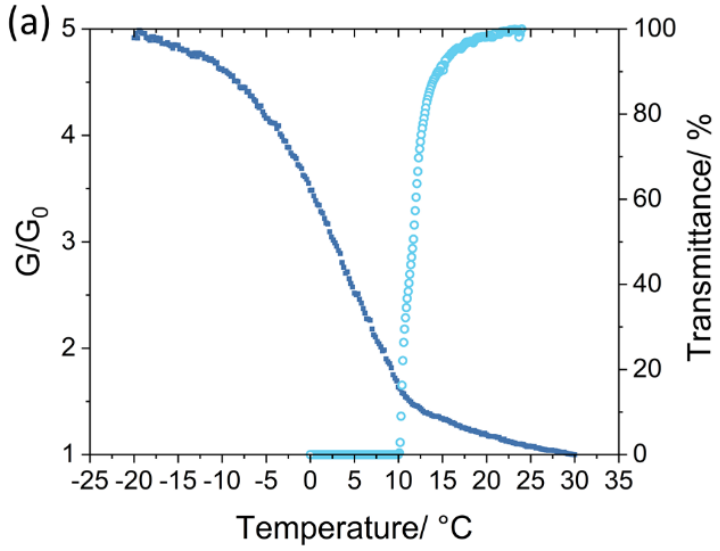

(b)
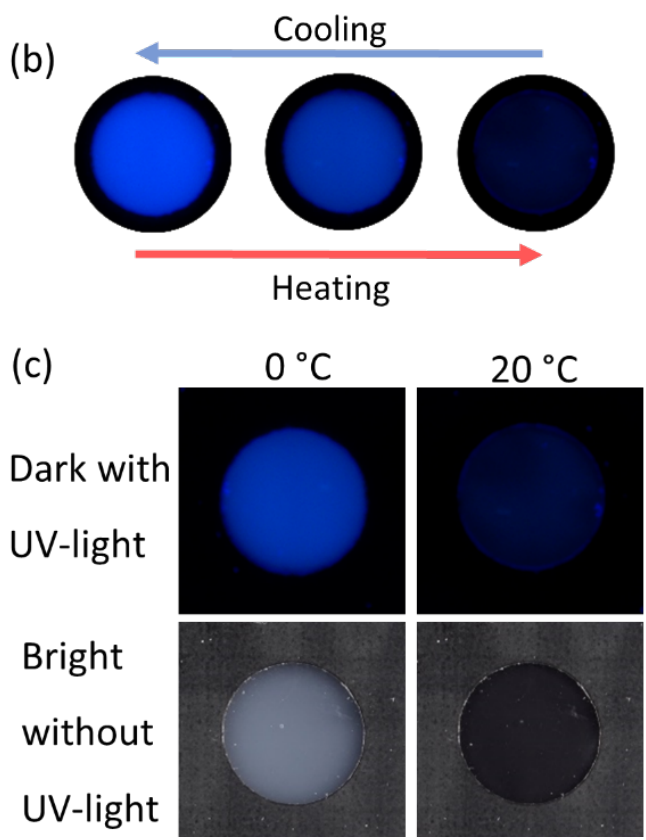

Figure 4 (a) Plot of Grey/Greyo (dark b ve squares) and ght transm tlance ( ght bue open cices) as a funct on of temperature $G G_{0}$ values were calculated rrom photograph $c$ mages of a protolvpe s gn conta $n \mathrm{ng}$ P(S-CO-TPEA)/DINCH

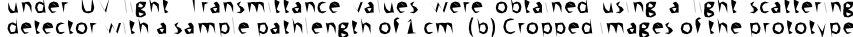
sign under $U$ ght, the temperature of the patch from eft to $\mathrm{ght}_{\mathrm{g}}-20^{\circ} \mathrm{C}, 0^{\circ} \mathrm{C}$ and $20^{\circ} \mathrm{C}$ (C) Cropoed mages of he P(S-CO-TPEA)/DINCH patch at 0 or $20^{\circ} \mathrm{C}$,

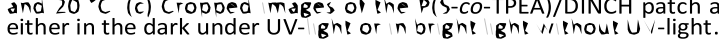

light and, importantly, used a UV cut-off filter for the camera, to only record the emitted light (at a higher wavelength) and not the back scattered light from the UV light source. A similar set-up, but without a UV cut-off filter, was used to measure polymer glass transition temperatures. ${ }^{67}$ The absorption and emission spectra for the $\mathrm{P}(\mathrm{S}-\mathrm{CO}-\mathrm{TPEA}) / \mathrm{DINCH}$ mixture at $2{ }^{\circ} \mathrm{C}$ is presented in Figure S12, with absorption and emission maxima at 348 and $472 \mathrm{~nm}$, respectively.

To quantify the luminescence of the P(S-CO-TPEA)/DINCH as a function of temperature a patch containing the mixture was cooled from 30 to $-20^{\circ} \mathrm{C}$, under UV light, and photos were taken every $0.2{ }^{\circ} \mathrm{C}$ (see also the video in ESI). These photos were converted to greyscale and the grey value of each was recorded (example image in Figure S13). The mean grey value was normalized to the lowest value and plotted against temperature in Figure 4a, dark blue squares. The increase in mean grey value as the patch cools can be divided into three phases. From 30 to 
$15^{\circ} \mathrm{C}$, the grey value gradually increases due to an increase in $\mathrm{FI}$ from the TPE side group. This emission increase is observed by flurospectroscopy (Figure S11). At this temperature range, the $\mathrm{P}(\mathrm{S}$-co-TPEA) chains are solvated and the increase in $\mathrm{Fl}$ is caused by both an increase in solvent viscosity and a decrease in thermal energy. The intermolecular rotations of AIE molecules have been shown to be susceptible to both increase in solvent viscosity at a fixed temperature and as a function of temperature in a solvent with a low viscosity index. ${ }^{41,48,68,69}$ At $11.5{ }^{\circ} \mathrm{C}$, an inflection point in the grey value and a linear increase down to $0^{\circ} \mathrm{C}$ is observed. At this temperature range, the $\mathrm{P}$ (S-Co-TPEA) chains begin to phase separate, as shown by a sharp decrease in light transmission (Figure 4a, light blue open circle) and a decrease in apparent $\mathrm{Fl}$, due to scattering (Figure S11). It is the collapse of chain restricted intermolecular motions of the TPE side chain group which leads to a greater increase in $\mathrm{Fl}$ in the region. This effect has been reported for the LCST phase separation of TPE-labelled PNIPAM, 51 TPE-PNIPAM chain decorated cholic acid showing UCST activity 57 and the collapse of TPE decorated poly(acrylic acid) chains in the presence of $\mathrm{Ca}^{2+}$ ions. ${ }^{70}$ From 0 to $-20^{\circ} \mathrm{C}$ the change in grey value decreases to the same gradient as 30 to $15{ }^{\circ} \mathrm{C}$. This signifies that the collapse of chains has reach a steady state and then only effect on the FI of TPE is the solvent viscosity and temperature of the system. This observation also matches the plateau of grey value seen in PS/DINCH at low temperatures (Figures $1 \mathrm{~b}$ and $\mathrm{S} 5$ ). In Figure S11, the $\mathrm{FI}$ also increases below 0 ${ }^{\circ} \mathrm{C}$, albeit a lower rate mostly due to reduced UV absorbance and emission due to the high opacity of the sample. The increase in fluorescence under UV-light is visualised in Figure $4 \mathrm{~b}$, cropped images of the patch are shown at $-20,0$ and $20^{\circ} \mathrm{C}$, from left to right. As for the PS/DINCH prototype signs, the P(Sco-TPEA)/DINCH sign also shows a contrast change in bright light without a fluorescent emission, as shown in Figure 4c.

\section{Conclusions}

We hope that our prototype thermotropic, electricity-free road signs demonstrate a working concept that could warn of the potential presence of ice to pedestrians or vehicles. Not only did we demonstrate that our manufactured signs clearly displayed opaque snowflake patterns, we also showed that, beside light scattering, a marked increase in fluorescence intensity was accomplished, for use in the dark. Although the fluorescent prototype sign presented shows significant potential, it not without its limitations. The fluorophore used has an absorbance maximum of $348 \mathrm{~nm}$, and although xenon car headlights have a broad emission spectrum, cerium-doped blubs ensure harmful UV radiation, $<400 \mathrm{~nm}$, is blocked. That withstanding, progress in expanding the library of AIE molecules continues and we hope our concept acts as a platform for future variations. The UCST-type response of the signs was fast ( $<60$ seconds) and shown to be reversible and stable over multiple cycles. The system can display a coloured thermo-response with the addition of solvent soluble dyes. Alternatively, a coloured transparent top layer could have been used to trigger a similar effect. Owing to the use of DINCH as the solvent, the warning sign can operate at high and sub-zero temperatures (Celsius scale), a difficultly faced by aqueous based systems. Although a switching temperature close to $0{ }^{\circ} \mathrm{C}$ was chosen to best illustrate the application's potential, this temperature was shown to be easily adjustable. At a fixed polymer/solvent composition the cloud point was easily changed by adjusting the molecular weight of the PS or through copolymerisation with (meth)acrylate or acrylamide comonomers. The phase separation of polymer blends with a large $T_{c p}$ difference displayed a two-step phase separation curve. Adjustment of cloud point allows for other applications, such as paint tins, warning users not to apply the coating onto surfaces below a certain temperature. The overall design philosophy of these prototype ice warning signs is aligned with an energy conscious and sustainable future and we hope they inspire a multitude of future optical applications.

\section{Author Contributions}

Joshua R. Booth: Conceptualization, data curation, formal analysis, investigation, methodology, project administration, validation, visualization, writing - original draft. Robert A. Young: Conceptualization, data curation, formal analysis, investigation, methodology, project administration, validation, visualization. Andrés N. Richards Gonzales: Formal analysis, investigation, validation, visualization. Zachary J. Meakin: Data curation, formal analysis, investigation, validation, visualization. Corinna M. Preuss: investigation, visualization. Ross W. Jaggers: investigation, visualization. Stefan A. F. Bon: Conceptualization, supervision, project administration, methodology, formal analysis, funding acquisition, writing review and editing.

\section{Conflicts of interest}

There are no conflicts to declare.

\section{Notes and references}

1 Road accidents and safety statistics, https://data.gov.uk/dataset/cb7ae6f0-4be6-4935-9277-

47e5ce24a11f/road-safety-data (accessed January 2021).

2 M. Rubinstein and R. H. Colby, in Polymer Physics, OUP Oxford, 2003, pp. 137-170.

3 M. L. Huggins, Annals of the New York Academy of Sciences, 1942, 43, 1-32.

4 P. J. Flory, J. Chem. Phys., 1941, 9, 660-660.

5 A. Seeboth, J. Schneider and A. Patzak, Solar Energy Materials and Solar Cells, 2000, 60, 263-277.

6 Y. Ke, C. Zhou, Y. Zhou, S. Wang, S. H. Chan and Y. Long, Advanced Functional Materials, 2018, 28, 1800113.

7 A. Seeboth and D. Lötzsch, Thermochromic and Thermotropic Materials, CRC Press, 2013.

8 S. Werner, H. J. Otto and U. Terbrack, DE Pat., DE3436477, 1986. 
9 W. Eck, H.-J. Cantow and V. Wittwer, DE Pat., DE4206317, 1993.

10 D. J. Zalucha, F. Sexsmith, D. D. Howard, M. L. Nulph, US Pat., 4409383, 1983

11 A. Beck, W. Körner, H. Scheller, J. Fricke, W. J. Platzer and V. Wittwer, Solar Energy Materials and Solar Cells, 1995, 36, 339347.

12 Y. Zhou, Y. Cai, X. Hu and Y. Long, J. Mater. Chem. A, 2014, 2, 13550-13555.

13 T.-G. La, X. Li, A. Kumar, Y. Fu, S. Yang and H.-J. Chung, ACS Appl. Mater. Interfaces, 2017, 9, 33100-33106.

14 F. Reinitzer, Monatshefte für Chemie, 1888, 9, 421-441.

15 A. Sasaki, K. Kurahashi and T. Takagi, Journal of Applied Physics, 1974, 45, 4356-4359.

16 N. A. Vaz and G. P. Montgomery, Journal of Applied Physics, 1987, 62, 3161-3172.

17 G. P. Montgomery and N. A. Vaz, Phys. Rev. A, 1989, 40, 6580-6591.

18 K. Resch and G. M. Wallner, Solar Energy Materials and Solar Cells, 2009, 93, 119-128.

19 V. Aseyev, H. Tenhu and F. M. Winnik, in Self Organized Nanostructures of Amphiphilic Block Copolymers II, eds. A. H. E. Müller and O. Borisov, Springer Berlin Heidelberg, 2010, vol. 242, pp. 29-89.

20 C. Zhao, Z. Ma and X. X. Zhu, Progress in Polymer Science, 2019, 90, 269-291.

21 L. Tang, L. Wang, X. Yang, Y. Feng, Y. Li and W. Feng, Progress in Materials Science, 2021, 115, 100702.

22 C. Pietsch, U. S. Schubert and R. Hoogenboom, Chem. Commun., 2011, 47, 8750-8765.

$23 \mathrm{~J}$. Seuring and S. Agarwal, Macromolecular Rapid Communications, 2012, 33, 1898-1920.

24 Q. Zhang and R. Hoogenboom, Progress in Polymer Science, 2015, 48, 122-142.

25 A. Imre and W. A. Van Hook, Journal of Physical and Chemical Reference Data, 1996, 25, 637-661.

26 A. Seeboth, H.-R. Holzbauer and R. Lange, Materialwissenschaft und Werkstofftechnik, 1998, 29, 336338.

27 M. Danko, Z. Kroneková, M. Mrlik, J. Osicka, A. bin Yousaf, A. Mihálová, J. Tkac and P. Kasak, Langmuir, 2019, 35, 13911403.

28 Y. Ding, Y. Yan, Q. Peng, B. Wang, Y. Xing, Z. Hua and Z. Wang, ACS Appl. Polym. Mater., 2020, 2, 3259-3266.

29 C. Pietsch, R. Hoogenboom and U. S. Schubert, Polym. Chem., 2010, 1, 1005-1008.

30 J. Szydlowski and W. A. Van Hook, Macromolecules, 1991, 24, 4883-4891.

31 S. Saeki, N. Kuwahara, S. Konno and M. Kaneko, Macromolecules, 1973, 6, 246-250.

32 S. Saeki, S. Konno, N. Kuwahara, M. Nakata and M. Kaneko, 1974, 7, 6.

33 S. Saeki, N. Kuwahara, S. Konno and M. Kaneko, Macromolecules, 1973, 6, 589-593.

34 A. Hartwig, H. Parlar and T. H. Brock, Air Monitoring Methods (The MAK-Collection for Occupational Health and
Safety, Part III: Air Monitoring Methods (DFG)), Wiley-VCH, 2013, vol. 13.

35 D. M. Price, Thermochimica Acta, 2001, 367-368, 253-262.

36 C. Rangel-Nafaile and J. J. Muoz-Lara, Chemical Engineering Communications, 1987, 53, 177-198.

37 P. Stepanek, C. Konak and B. Sedlacek, Macromolecules, 1982, 15, 1214-1216.

38 F. Tao, J. Han, Q. Gu, C. Teng, D. Zou, D. Zhou and G. Xue, Macromolecules, 2008, 41, 9890-9895.

39 E. E. Jelley, Nature, 1936, 138, 1009-1010.

40 F. Würthner, T. E. Kaiser and C. R. Saha-Möller, Angew Chem Int Ed Engl, 2011, 50, 3376-3410.

41 P. F. Barbara, S. D. Rand and P. M. Rentzepis, J. Am. Chem. Soc., 1981, 103, 2156-2162.

42 W. J. Leigh and D. R. Arnold, J. Chem. Soc., Chem. Commun., 1980, 406-408.

43 M. A. El-Bayoumi and F. M. A. Halim, J. Chem. Phys., 1968, 48, 2536-2541.

44 J. Saltiel, O. C. Zafiriou, E. D. Megarity and A. A. Lamola, J. Am. Chem. Soc., 1968, 90, 4759-4760.

45 G. Fischer, G. Seger, K. A. Muszkat and E. Fischer, J. Chem. Soc., Perkin Trans. 2, 1975, 1569-1576.

46 K. Sandros, M. Sundahl, O. Wennerstroem and U. Norinder, J. Am. Chem. Soc., 1990, 112, 3082-3086.

47 J. Luo, Z. Xie, J. W. Y. Lam, L. Cheng, H. Chen, C. Qiu, H. S. Kwok, X. Zhan, Y. Liu, D. Zhu and B. Z. Tang, Chem. Commun., 2001, 1740-1741.

48 J. Chen, C. C. W. Law, J. W. Y. Lam, Y. Dong, S. M. F. Lo, I. D. Williams, D. Zhu and B. Z. Tang, Chem. Mater., 2003, 15, 15351546.

49 J. Mei, Y. Hong, J. W. Y. Lam, A. Qin, Y. Tang and B. Z. Tang, Advanced Materials, 2014, 26, 5429-5479.

50 A. Qin, J. W. Y. Lam and B. Z. Tang, Progress in Polymer Science, 2012, 37, 182-209.

51 L. Tang, J. K. Jin, A. Qin, W. Z. Yuan, Y. Mao, J. Mei, J. Z. Sun and B. Z. Tang, Chem. Commun., 2009, 4974-4976.

52 H. Zhou, F. Liu, X. Wang, H. Yan, J. Song, Q. Ye, B. Z. Tang and J. Xu, J. Mater. Chem. C, 2015, 3, 5490-5498.

53 T. Li, S. He, J. Qu, H. Wu, S. Wu, Z. Zhao, A. Qin, R. Hu and B. Z. Tang, J. Mater. Chem. C, 2016, 4, 2964-2970.

54 Z. Wang, T.-Y. Yong, J. Wan, Z.-H. Li, H. Zhao, Y. Zhao, L. Gan, X.-L. Yang, H.-B. Xu and C. Zhang, ACS Appl. Mater. Interfaces, 2015, 7, 3420-3425.

55 X. Yin, F. Meng and L. Wang, J. Mater. Chem. C, 2013, 1, 6767-6773.

56 X. Wang, K. Xu, H. Yao, L. Chang, Y. Wang, W. Li, Y. Zhao and J. Qin, Polym. Chem., 2018, 9, 5002-5013.

57 Y.-G. Jia, K.-F. Chen, M. Gao, S. Liu, J. Wang, X. Chen, L. Wang, Y. Chen, W. Song, H. Zhang, L. Ren, X.-X. Zhu and B. Z. Tang, Sci. China Chem., 2021, 64, 403-407.

58 Q. Li, X. Li, Z. Wu, Y. Sun, J. Fang and D. Chen, Polym. Chem., 2018, 9, 4150-4160.

59 P. J. Flory, Principles of Polymer Chemistry, Cornell University Press, 1953.

60 U. Heudorf, V. Mersch-Sundermann and J. Angerer, International Journal of Hygiene and Environmental Health, 2007, 210, 623-634. 
61 S. Benjamin, E. Masai, N. Kamimura, K. Takahashi, R. C. Anderson and P. A. Faisal, Journal of Hazardous Materials, 2017, 340, 360-383.

62 J. Yang, Z. Sun, W. Jiang and L. An, J. Chem. Phys., 2002, 116, 5892-5900.

63 H. E. Yang and Y. Chan Bae, Fluid Phase Equilibria, 2016, 417, 220-228.

64 T. Otsu, T. Ito, T. Fukumizu and M. Imoto, BCSJ, 1966, 39, 2257-2260.

65 V. D. Braun and G. Mott, Die Angewandte Makromolekulare Chemie, 1971, 18, 183-194.

66 Y. Shiraishi, R. Miyamoto, X. Zhang and T. Hirai, Org. Lett., 2007, 9, 3921-3924.

67 Z. Qiu, E. K. K. Chu, M. Jiang, C. Gui, N. Xie, W. Qin, P. Alam, R. T. K. Kwok, J. W. Y. Lam and B. Z. Tang, Macromolecules, 2017, 50, 7620-7627.

68 N. L. C. Leung, N. Xie, W. Yuan, Y. Liu, Q. Wu, Q. Peng, Q. Miao, J. W. Y. Lam and B. Z. Tang, Chemistry - A European Journal, 2014, 20, 15349-15353.

69 Y. Cai, L. Du, K. Samedov, X. Gu, F. Qi, H. H. Y. Sung, B. O. Patrick, Z. Yan, X. Jiang, H. Zhang, J. W. Y. Lam, I. D. Williams, D. L. Phillips, A. Qin and B. Z. Tang, Chem. Sci., 2018, 9, 4662-4670. 70 K. Morishima, F. Ishiwari, S. Matsumura, T. Fukushima and M. Shibayama, Macromolecules, 2017, 50, 5940-5945. 